\title{
Memory instruction interacts with both visual and motoric inhibition of return
}

\author{
Kate M. Thompson • Tracy L. Taylor
}

Published online: 17 January 2015

(C) The Psychonomic Society, Inc. 2014

\begin{abstract}
In the item-method directed forgetting paradigm, the magnitude of inhibition of return (IOR) is larger after an instruction to forget (F) than after an instruction to remember (R). In the present experiments, we further investigated this increased magnitude of IOR after $F$ than after R memory instructions, to determine whether this F $>$ R IOR pattern occurs only for the motoric form of IOR, as predicted, or also for the visual form. In three experiments, words were presented in one of two peripheral locations, followed by either an $\mathrm{F}$ or an R memory instruction. Then, a target appeared either at the same location as the previous word or at the other location. In Experiment 1, participants maintained fixation throughout the trial until the target appeared, at which point they made a saccade to the target. In Experiment 2, they maintained fixation throughout the entire trial and made a manual localization response to the target. The F> R IOR difference in reaction times occurred for both the saccadic and manual responses, suggesting that memory instructions modify both motoric and visual forms of IOR. In Experiment 3, participants made a perceptual discrimination response to report the identity of a target while the eyes remained fixed. The F $>$ R IOR difference also occurred for these manual discrimination responses, increasing our confidence that memory instructions modify the visual form of IOR. We relate our findings to postulated differences in attentional withdrawal following $\mathrm{F}$ and $\mathrm{R}$ instructions and consider the implications of the findings for successful forgetting.
\end{abstract}

K. M. Thompson $(\bowtie) \cdot$ T. L. Taylor

Department of Psychology, Dalhousie University, Halifax, Nova

Scotia, Canada

e-mail: thompskm@dal.ca
Keywords Attention-memory interaction - Inhibition of return

Our ability to learn from and remember characteristics of our environment is, arguably, one of the key factors underlying the sophistication of human functioning. Not only does memory provide us with a sense of self and continuity through time (Gallagher, 2000), but information from long-term memory influences even the most basic cognitive functions, such as perception and attention - this is at the heart of the wellknown interactions between top-down and bottom-up processing (e.g., Ciaramelli, Grady, \& Moscovitch, 2008; Duncan \& Humphreys, 1989; Posner \& Petersen, 1990).

In the study of memory, it is clear that forgetting irrelevant information that might otherwise interfere with successful encoding or retrieval can be just as important for creating an accurate representation of the world as remembering relevant information (MacLeod, 1998). For example, it serves us well to forget an instructional error made by a professor. If we were unable to forget such irrelevant information, it might interfere with our memory for the accurate information (Anderson, Bjork, \& Bjork, 1994; Anderson \& Neely, 1996; Postman \& Underwood, 1973). The intentional forgetting of irrelevant or misleading information is studied in the laboratory using the directed forgetting paradigm.

In this paradigm, participants are presented with information (typically words, but a wide variety of stimuli have been used-see, e.g., Hourihan, Ozubko, \& MacLeod, 2009; Quinlan, Taylor, \& Fawcett, 2010) and are asked to remember some things and to forget others. Two main procedures can be used: the list method and the item method. The present investigation concerns the item method (for reviews of both methods, see MacLeod, 1998, or Basden \& Basden, 1998). 
Participants in an item-method directed forgetting paradigm are presented with items one at a time, and each is followed by an instruction to remember (R) or forget (F). After all items have been presented, participants' memory of both $\mathrm{R}$ and $\mathrm{F}$ items is tested with some kind of explicit test of memory (often yes-no recognition; although see Thompson, Fawcett, $\&$ Taylor, 2011). The typical results show greater memory performance for $\mathrm{R}$ items than for $\mathrm{F}$ items - the directed forgetting effect (DF effect). Researchers are confident that this effect is not simply the result of demand characteristics on the part of participants (MacLeod, 1999).

The main explanation of the DF effect, the selective rehearsal hypothesis, posits that better memory for $\mathrm{R}$ than for $\mathrm{F}$ items is achieved primarily by selective elaborative rehearsal of $\mathrm{R}$ items over F items (e.g., Bjork \& Woodward, 1973; MacLeod, 1975; Woodward, Bjork, \& Jongeward, 1973). Whereas $\mathrm{R}$ items are afforded as much distinctive processing as possible to ensure that they are encoded, the processing of $\mathrm{F}$ items stops when the $\mathrm{F}$ instruction is received, to limit the transfer of this information to memory.

Interestingly, much evidence suggests that, rather than passively dropping $\mathrm{F}$ items from working memory, instantiation of an instruction to forget is achieved by an active, cognitively effortful process (Bastin et al., 2012; Fawcett \& Taylor, 2008; Nowicka, Marchewka, Jednorog, Tacikowski, \& Brechmann, 2011; Saletin, Goldstein, \& Walker, 2011; Wylie, Foxe, \& Taylor, 2008). Relevant to the present experiment, a substantial amount of research has investigated inhibition of return (IOR) in item-method directed forgetting tasks, which has informed our understanding of the cognitive consequences of instantiating an instruction to forget (Fawcett \& Taylor, 2010; Taylor, 2005; Taylor \& Fawcett, 2011; Thompson, Hamm, \& Taylor, 2014). As will be described below, instantiating memory instructions at encoding produces interactions with IOR that implicate a differential withdrawal of attentional resources from $\mathrm{F}$ versus $\mathrm{R}$ items (see Taylor, 2005; Taylor \& Fawcett, 2011). The fact that this interaction seems specific to a motor form of IOR further suggests that the allocation of limited-capacity attentional resources during encoding not only determines the contents of memory in the long term, but also influences subsequent information processing in the short term. In this way, limiting the further encoding of unwanted or irrelevant items in working memory invokes a complex interplay of attentional, memorial, and motor systems.

\section{Inhibition of return}

In an IOR cueing paradigm, participants are presented with an uninformative visual cue to the left or right (e.g., the brightening of an outline box) that they are instructed to ignore. This cue is followed by a target to the left or right that requires a speeded response. If the stimulus onset asynchrony (SOA) between the cue and the target is relatively short (less than $\sim 300 \mathrm{~ms}$ ), reaction times (RTs) to respond to the target are faster at the cued location than at the other, uncued location. This facilitatory effect for target RTs occurs because the cue automatically draws attention to it, and this attention increases the speed and efficiency of visual processing (Posner, 1980). However, if the target is presented at a longer SOA (more than $\sim 300 \mathrm{~ms}$ ), RTs are slower at the cued than at the uncued location. The latter pattern is known as IOR (Posner \& Cohen, 1984). Critically, IOR is observed in RTs only after attention has been withdrawn from the cued location; it is otherwise masked by the opposing facilitatory effects of attentional capture (Danziger \& Kingstone, 1999).

IOR can be understood as reflecting a mechanism that promotes efficient search strategies by decreasing the likelihood that a previously inspected location will be reinspected (Klein, 2000; Klein \& MacInnes, 1999; MacInnes \& Klein, 2003). IOR is initiated by the activation of the oculomotor system by a stimulus (Rafal, Calabresi, Brennan, \& Sciolto, 1989; Taylor \& Klein, 1998; but see Chica, Klein, Rafal, \& Hopfinger, 2010), and/or by modulations of mental spatial saliency maps after attention is withdrawn from a noninformative cue (Henderickx et al., 2012). Critically, though, the subsequent effects on information processing (i.e., the particular kinds of processing that are slowed at the cued relative to the uncued location) vary depending on the state of the oculomotor system. When the oculomotor system is active (i.e., eye movements - or saccades - are allowed/ required to the cue and/or target), IOR manifests as a motoric bias against responding toward the cued location. Conversely, when the oculomotor system is suppressed (i.e., saccades are prevented during the task by requiring that participants maintain fixation in one location), IOR manifests as a perceptual deficit for information presented in the cued location (Chica, Taylor, Lupiáñez, \& Klein, 2010; Hilchey, Klein, \& Ivanoff, 2012; Hunt \& Kingstone, 2003; Taylor \& Klein, 2000). These two forms of IOR are dubbed motoric and visual, respectively (Taylor \& Klein, 2000). Importantly, these two forms of IOR do not co-occur in behavior (Chica et al., 2010b; Hilchey et al., 2012; Taylor \& Klein, 2000). For example, Hilchey et al. found that the magnitude of IOR was the same when participants are required to make saccades regardless of whether the cues and targets were presented centrally or peripherally. If the motoric and visual forms co-occurred, it would be expected that the magnitude of IOR would be greater when saccades were made to peripheral cues (this type of task involves a motoric component - the saccadeas well as a perceptual component - the stimulation of the target location by a peripheral cue). Similarly, Chica, et al. found that typical visual IOR effects are not observed when participants are required to make saccades to cues or targets. Finally, in investigations of IOR using event-related potential 
(ERP) technology, reductions in P1 (an early sensory component) occur under conditions that elicit the motoric as well as those that elicit the visual form of IOR. However, these P1 modulations correlate with behavior only when the oculomotor system is suppressed (Satel, Hilchey, Wang, Story, \& Klein, 2013). The distinction between these two forms of IOR is also supported by neurophysiological evidence that they are differentially affected by brain damage and rTMS manipulations, in which double dissociations have been observed (Bourgeois, Chica, Migliaccio, Thiebault de Schotten, \& Bartolomeo, 2012; Bourgeois, Chica, Valero-Cabré, \& Bartolomeo, 2013).

\section{IOR in item-method directed forgetting}

Taylor (2005) first investigated IOR in item-method directed forgetting by creating a directed forgetting cueing paradigm. In this paradigm, participants were presented with a word to the left or right (serving as the "cue" that initially draws attention) followed by an auditory R or F memory instruction. Then, after a relatively long $\mathrm{SOA}(1,200 \mathrm{~ms}$ from word onset) a target dot was presented to the left or right, which participants localized using a manual button-press. Taylor found that the magnitude of IOR (RT to "cued" targets- RT to "uncued" targets) was greater after an $\mathrm{F}$ than after an $\mathrm{R}$ instruction $(\mathrm{F}>\mathrm{R}$ IOR). Because IOR appears in RTs after attention has been withdrawn from the cued location (Danziger \& Kingstone, 1999), the relative magnification of IOR by an $F$ instruction was interpreted as a more ready withdrawal of attention following $\mathrm{F}$ instructions than following $\mathrm{R}$ instructions. This explanation converges with demonstrations that instantiating a forget instruction is relatively more cognitively demanding than instantiating a remember instruction (Fawcett \& Taylor, 2008) and engages frontal mechanisms implicated in executive control over attention (Bastin et al., 2012; Nowicka et al., 2011; Saletin et al., 2011; Wylie et al., 2008). The differential withdrawal of attention following $\mathrm{F}$ and $\mathrm{R}$ instructions also accounts for the fact that these instructions impact processing of subsequent task-irrelevant information that appears in close spatial and temporal proximity to the study item (Fawcett \& Taylor, 2012). Importantly, when the F instructions occur after the disappearance of the study items, there is no evidence for the reorienting of processing resources to the opposite location (Taylor \& Fawcett, 2012); this establishes that the F $>$ R IOR pattern does, in fact, reflect relative magnification of IOR by an $\mathrm{F}$ instruction (i.e., rather than being due to attentional facilitation at the opposite location; see also Thompson et al., 2014). Thus, participants actively withdraw their attention from $\mathrm{F}$ items, and this active process may be partially responsible for successful intentional forgetting. However, IOR is a complex phenomenon, and further investigation was necessary to determine specifically what kinds of processing might be shared between intentional forgetting and IOR that would result in their interaction. In particular, investigating whether memory instruction interacts with both the motoric and visual forms of IOR should elucidate which specific mechanisms (motoric or perceptual) are associated with intentional forgetting.

Taylor and Fawcett (2011) replicated Taylor's (2005) methodology, but, in two conditions relevant to the present study, had participants make either a spatially compatible localization response (button-press on the left for a target that appeared on the left, button-press on the right for a target that appeared on the right), or a perceptual discrimination response (one button-press to report the identity of a target as an upright triangle, a different button-press to report the identity as an inverted triangle). The F $>$ R IOR difference emerged only when participants localized the target, not when they reported its identity. The spatial localization response required that a response be made toward the location of a target, whereas the perceptual discrimination response required an analysis of the perceptual quality of the target. As we described above, depending on the state of the oculomotor system, IOR may manifest as either a bias against responding to targets that arise at the cued location (the motoric form of IOR) or as impaired/delayed perception of information at the cued location (the visual form). Because the interaction between memory instruction and IOR was observed only with a localization response, Taylor and Fawcett (2011) concluded that the interaction was specific to the motoric form of IOR. They presumed that selective enhancement of the motoric form of IOR by an $\mathrm{F}$ instruction could indicate a bias against responding to a source of unreliable information (see Thompson et al., 2014, for additional support for this hypothesis). This reluctance to respond to information arising from the same location as previous misinformation suggests that instructions to forget impact not only the encoding of to-be-forgotten items, but also subsequent information processing. To the extent that episodic memory keeps a record of goal-directed behavior (Conway, 2009), an alteration in behavior due to instantiation of an encoding instruction might provide a means for an encoding instruction to influence memory not only for the instructed item itself, but for the larger episodic event in which the item is embedded. In other words, an instruction to forget might impair episodic memory directly by limiting the encoding of the $\mathrm{F}$ item, and indirectly by altering the subsequent goaldirected behavior that defines the episode for which the trace is established.

\section{The present experiments}

There has been one potentially critical oversight in the investigation of the $\mathrm{F}>\mathrm{R}$ IOR difference that warrants some attention. In a typical cueing paradigm designed to differentiate 
between the motoric and visual forms of IOR, an important methodological component is the restriction and monitoring of participants' eye movements. Motoric IOR is observed when the oculomotor system is active, and visual IOR is observed when the oculomotor system is suppressed. Critically, Chica et al. (2010b ) showed that suppression of the oculomotor system is necessary to observe the visual form of IOR. In their experiment, they had participants perform a detection task or a color discrimination task. IOR was observed in both tasks when the oculomotor system was suppressed by preventing eye movements, suggesting an effect on perceptual processing, consistent with visual IOR. However, when the oculomotor system was activated by having participants make eye movements, IOR was only observed in the detection task. This shows that the visual form of IOR (as measured by IOR in the color discrimination task) is only observed in RTs when the oculomotor system is suppressed, and suppression can only be guaranteed by monitoring participant's eye movements. In addition, Hilchey et al. (2012; see also Taylor \& Klein, 2000) found that perceptual and motoric effects on RT are not additive in a motoric IOR task by showing equivalent magnitudes of IOR for both central arrow targets (which could only measure a motoric bias) and peripheral targets (which could measure a motoric bias and perceptual degradation).

As we described above, Taylor and Fawcett (2011) reported no $\mathrm{F}>\mathrm{R}$ IOR when participants made a perceptual discrimination response to the target. They interpreted this as evidence that memory instruction does not modulate visual IOR effects, and that the interaction was due to an increased bias (like the one responsible for motoric IOR) against responding toward the source of irrelevant information. However, it is likely that participants were moving their eyes to fixate the study words on each trial in order to read them in Taylor and Fawcett (2011). According to Rayner (1998), the human perceptual span ranges from 3-4 letters on the left of fixation to 14-15 letters on the right of fixation. At the viewing distance and font size used by Rayner, this corresponds to about 1 deg of visual angle on the left, and 4-5 deg on the right. In addition, Rayner notes that the identification span (i.e., the distance at which words can be identified) is even smaller, at 7-8 letters to the right, or about 2 deg of visual angle. In previous investigations of IOR in directed forgetting, the minimum distance from fixation to the boundaries of the peripheral locations at which words were presented was $4.5 \mathrm{deg}$ of visual angle (Fawcett \& Taylor, 2010; Taylor, 2005; Taylor \& Fawcett, 2011; Thompson et al., 2014). Words are typically centered in either the left or right peripheral location, thus the last (if presented to the left) or first (if presented to the right) letter of each word would be no closer than $4.5 \mathrm{deg}$ of visual angle from fixation. This, in combination with the fact that participants were never instructed to refrain from moving their eyes from fixation, means that participants were almost certainly moving their eyes to read the words, even during the perceptual discrimination task in Taylor and Fawcett (2011). Given that activation of the oculomotor system may mask, override, hide, or cancel any visual IOR effects that would otherwise occur if the oculomotor system were suppressed (Chica et al., 2010b), it is unsurprising that the visual IOR effects that Taylor and Fawcett (2011) were testing for might have been masked (the 8-ms overall IOR effect they observed in their discrimination task was only marginally significant and did not interact with memory instruction). Thus, a more controlled test of the effects of memory instruction on motoric and visual forms of IOR is needed, and our understanding of the nature of this interaction hinges upon such a test.

The present experiments directly assessed whether the F> R IOR difference represents a selective modulation of the motoric form of IOR, as hypothesized by Taylor and Fawcett (2011) and supported by Thompson et al. (2014), or whether the explicit restriction of eye movements will reveal modulation of visual IOR as well. If, under controlled conditions and careful eye movement monitoring, we observe an interaction between memory instruction and visual IOR, it will challenge the previous conclusions about the mechanisms and implications of the F> R IOR difference. Participants completed a directed forgetting cueing paradigm similar to that used in previous investigations of IOR in directed forgetting. On each trial, a word was presented to the left or right of a central fixation and was followed by an auditory $\mathrm{R}$ or $\mathrm{F}$ memory instruction. After a relatively long SOA relative to the word, a target appeared to the left or right. In Experiment 1, participants were required to maintain fixation at the center of the screen until making a saccade to the target. In Experiment 2, participants were required to maintain fixation at center throughout the entire trial, and make a manual spatially compatible localization response to the target. Participants' eye movements were monitored with an eye tracker in both experiments. This ensured that the participants were adhering to the fixation/saccade requirements of their condition. Because the oculomotor system should be engaged in Experiment 1, any IOR observed in that experiment should be motoric in nature. IOR in Experiment 2 should be visual in nature since participants are required to suppress the oculomotor system in this experiment (Chica et al., 2010b; Hilchey et al., 2012; Hunt \& Kingstone, 2003; Taylor \& Klein, 2000). Thus, if the interaction of memory instructions and IOR is due to selective modulation of the motoric form of IOR, the F> R IOR pattern should be observed in Experiment 1 only. If, however, memory instructions also interact with the visual form of IOR, the F> R IOR pattern should be observed in Experiment 2, as well. 


\section{Experiment 1}

In Experiment 1, participants were presented with study words one at a time to the left or right of central fixation, each followed by an auditory R or F memory instruction, and then by a target in either the same location as the word or the opposite location. Participants maintained fixation until the target appeared, at which point they moved their eyes to the target. Because the oculomotor system was activated by this requirement to fixate the target, the IOR in this experiment should be motoric in nature (Chica et al., 2010b; Hilchey et al., 2012; Hunt \& Kingstone, 2003; Taylor \& Klein, 2000). To reiterate our predictions: If memory instruction interacts with motoric IOR as it has in previous directed forgetting cueing experiments, the $\mathrm{F}>\mathrm{R}$ IOR pattern should occur in this experiment.

\section{Method}

\section{Participants}

Twenty-nine participants were recruited from the undergraduate subject pool at Dalhousie University and received one credit point for participating. All of the participants reported normal or corrected-to-normal vision and a good understanding of the English language. The experiment was approved by the Human Research Ethics board at Dalhousie University, and thus meets the ethical standards set forth in the TriCouncil Policy Statement.

\section{Materials}

For the experiment, we used SR Research Experiment Builder Version 1.10.1 on an Intel Core 2 computer running Microsoft Windows XP Professional Version 2002. The stimuli were presented on a 32 -in. 1,366 $\times 768$ resolution Phillips LCD monitor (Model ID: BDL3231C/00). Participants viewed the monitor from a distance of approximately $55 \mathrm{~cm}$. Eye position was monitored with an EyeLink II (version 2.21) eyetracking system.

A master word list of 320 nouns was selected from the Paivio, Yuille, and Madigan Word Pool using an online generator (www.math.yorku.ca/SCS/Online/paivio/). The words had a mean Kučera-Francis word frequency of 47.3 (ranging from 0 to $100, S D=36.7$ ), a mean imagery rating of 5.4 (ranging from 2 to $6.9, S D=1.3$ ), and a mean concreteness rating of 5.4 (ranging from 1.2 to $7, S D=1.8$ ). Words ranged in length from three to six letters $(M=5, S D=0.9)$. For each participant, the Experiment Builder software randomized this word list and split it into four lists of $20 \mathrm{R}$ words, four lists of $20 \mathrm{~F}$ words, and a list of 160 Foil words.
Each trial in the study phase began with the presentation of a centrally located fixation stimulus (+; Arial size 18 font) and two circular gray placeholders on a black background. Each placeholder measured $1 \mathrm{deg}$ of visual angle. One placeholder was centered $3.5 \mathrm{deg}$ to the right of fixation, and the other was centered $3.5 \mathrm{deg}$ to the left. Words were presented in Arial bold, size 14 font in yellow text, replacing one of the gray placeholders. Yellow circles (of the same size and eccentricity as the gray placeholders) served as the targets. In studies that have used a similar paradigm, the cue and target were also both the same color, but were black on a white background instead of yellow on a black background (e.g., Taylor, 2005; Taylor \& Fawcett, 2011). Our use of yellow for both the word and target was motivated by the fact that pilot testing had shown yellow to be more visible on the background than white - an important consideration when peripheral words had to be read while the eyes remained fixed at the center. We have no reason to believe that having the word and target both in yellow, rather than both in black, would have had any impact on our findings. An intertrial fixation stimulus was visible in the center of the screen between trials and was used for drift correction before each trial. This stimulus was a white circle measuring $1 \mathrm{deg}$, with a $0.4-\mathrm{deg}$ black circle in its center.

Two auditory tones, one relatively high-pitched $(1170 \mathrm{~Hz})$ and one relatively low-pitched $(260 \mathrm{~Hz})$, were used as the memory instructions. The assignment of memory instructions to tones was counterbalanced, such that half of the participants were told that the high-pitched tone was an $\mathrm{R}$ instruction and the low-pitched tone an F instruction, whereas the other half of the participants were told the opposite (low tone $=\mathrm{R}$, high tone $=\mathrm{F})$.

\section{Procedure}

Participants were given verbal instructions detailing the task, along with a visual depiction of the trial progression in the study phase. Participants were informed that they were to do their best to follow the memory instruction for each word, and that they were to respond to all targets as quickly and accurately as possible. Participants were told that the study phase would be followed by a memory test, but they were not told that they would be tested for their memory of $\mathrm{F}$ items as well.

Study phase There were $20 \mathrm{R}$ items and $20 \mathrm{~F}$ items for each type of trial, for a total of 160 trials in the study phase. A depiction of a trial is presented in Fig. 1. Each trial was initiated by the participant by depressing the space bar while maintaining fixation on the intertrial fixation stimulus. This button-press initiated a drift correction before each trial, and then initiated the trial once drift correction was complete. Upon initiation of the trial, a fixation cross ("+") replaced the intertrial fixation stimulus, and two circular gray 


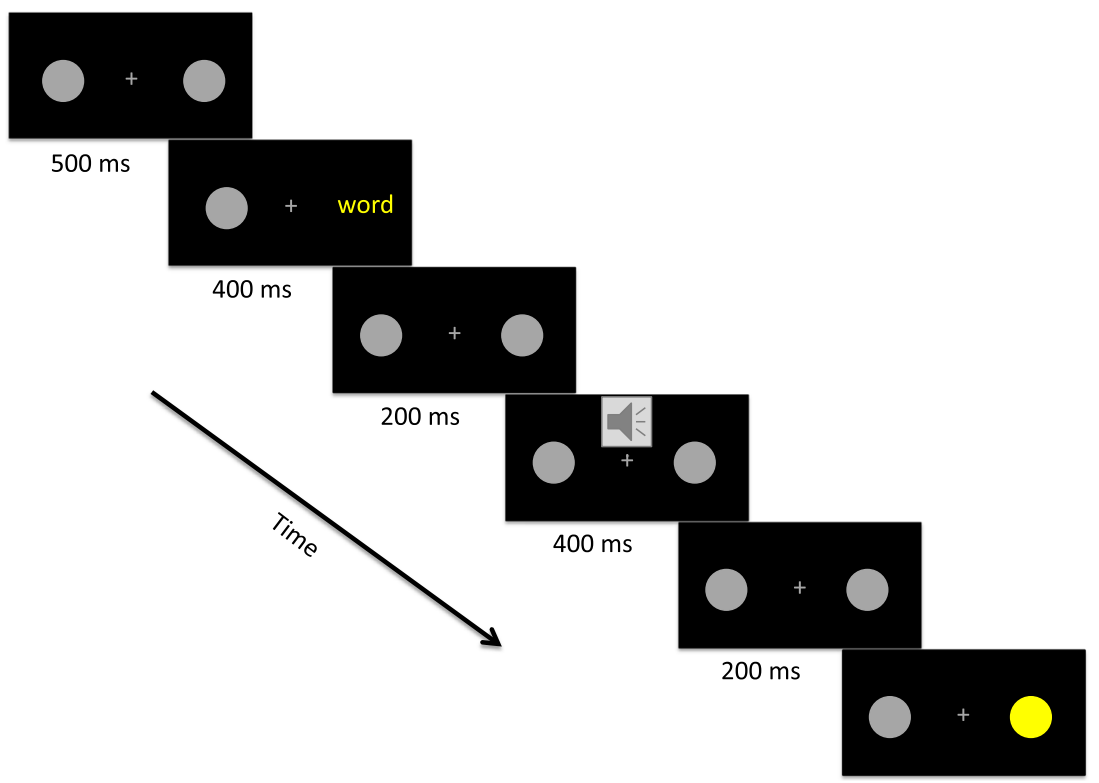

Fig. 1 Progression of a trial in Experiments 1 and 2

placeholders (one to the right and one to the left of fixation) appeared. A word replaced one of the placeholders $500 \mathrm{~ms}$ after the start of the trial. The word was equally likely to appear in the place of the right or the left placeholder, and it remained visible for $400 \mathrm{~ms}$. The placeholder reappeared upon word disappearance. An auditory R or F memory instruction (high- or low-pitched tone) was presented $200 \mathrm{~ms}$ after the disappearance of the word and lasted $400 \mathrm{~ms}$. A target (yellow circle) replaced one of the placeholders $200 \mathrm{~ms}$ after the end of the memory instruction. The target was equally likely to appear in the place of the right or left placeholder, and it remained visible for 1,000 ms. Participants were given 2, $000 \mathrm{~ms}$ from the onset of the target to make a response. RT and accuracy were measured. Participants were told to maintain fixation at the center until the target appeared, at which point they should move their eyes from fixation to the target (i.e., make a saccade to the target location) as quickly as they could. If any erroneous eye movements (saccades ending more than $2.5 \mathrm{deg}$ away from the central fixation ${ }^{1}$ ) were detected before the target appeared, the trial in progress was aborted. Participants were tested for their memory of the words presented on aborted trials, but these words were excluded from the analyses of our results.

Recognition phase After all study trials had been presented, participants completed a yes-no recognition task. All R and F items from the study phase were presented, along with an equal number of foil items. Thus, 160 study words plus 160 unstudied foil words were presented randomly, making a total of 320 trials in the recognition phase. All words were

\footnotetext{
${ }^{1}$ This eccentricity is similar to what has been used in other studies of IOR (see, e.g., Hilchey et al., 2012).
}

presented centrally on the computer monitor one at a time. Participants were to indicate whether they recognized the word as having appeared during the study phase, regardless of the memory instruction they had received at study. If they recognized the word, they were told to press the " $y$ " button, and if they did not, they were told to press the " $n$ " button. After all study and foil words had been presented, participants were debriefed and had any questions answered by the experimenter.

\section{Results}

Proportion of retained study trials Study trials were retained for analysis only if participants refrained from making eye movements before target onset. To determine whether study trials were retained differentially across conditions, a 2 (WordTarget Location: same, different) $\times 2$ (Memory Instruction: R, F) repeated measures analysis of variance (ANOVA) was conducted with the proportions of retained trials as the dependent measure. There were no significant effects or interactions (all $F \mathrm{~s}<1$ ). Thus, participants' ability to follow the fixation instructions was not influenced by the type of trial that was presented (see Table 1 for descriptive statistics).

Recognition accuracy Although words from aborted study trials were tested during the recognition phase, they were excluded from the calculations of recognition accuracy. The data from the recognition test were analyzed using a one-way repeated measures ANOVA with word type (R, F, foil) as the independent variable and the proportion of "yes" responses as the dependent variable. We found a significant main effect of 
Table 1 Descriptive statistics of the proportions of retained study trials per condition in Experiment 1. Means are reported, with standard deviations in parentheses

\begin{tabular}{lll}
\hline & \multicolumn{2}{l}{ Word-Target Location } \\
\cline { 2 - 3 } Memory Instruction & Same & Different \\
\hline Remember & $.59(.12)$ & $.60(.14)$ \\
Forget & $.57(.14)$ & $.60(.13)$ \\
\hline
\end{tabular}

word type $[F(2,56)=62.847, M S E=.011, p<.001]$ such that $\mathrm{R}$ items $(M=.51)$ were recognized at a higher rate than $\mathrm{F}$ items $(M=.37)[t(28)=6.475, p<.001]$. This is the expected $\mathrm{DF}$ effect (better memory for $\mathrm{R}$ than for $\mathrm{F}$ items). Both $\mathrm{R}$ and $\mathrm{F}$ items were recognized at a higher rate than foil words $(M=$ .20) $[t(28)=9.167, p<.001$, and $t(28)=6.474, p<.001$, respectively]. These results confirmed that participants had used the memory instructions as intended at study.

Saccadic RTs Given that participants had used the memory instructions as intended, the key question was whether these instructions interacted with the IOR effect measured by saccadic RTs to the targets presented at study. See Fig. 2 for descriptive statistics. A 2 (Word-Target Location: same, different $) \times 2$ (Memory Instruction: R, F) repeated measures ANOVA was conducted on the saccadic RTs to the targets. A significant main effect of word-target location was apparent $[F(1,28)=36.590, M S E=1,366.332, p<.001]$, with slower RTs to targets in the same location as the previous word than to those in the other location (an IOR effect). There was also a significant main effect of memory instruction $[F(1,28)=$ $12.157, M S E=760.716, p=.002]$, with slower RTs after $\mathrm{F}$ than after R instructions. Critically, a significant Word-Target Location $\times$ Memory Instruction interaction influenced the RTs $[F(1,28)=4.510, M S E=826.604, p=.043]$. The interaction was due to the fact that the magnitude of IOR (same RTdifferent $\mathrm{RT})$ was greater after $\mathrm{F}(M=52.871 \mathrm{~ms})$ than after $\mathrm{R}$ $(M=30.169 \mathrm{~ms})$ instructions.

Analogous analyses on the accuracy of target responses yielded no significant effects (all $F_{\mathrm{S}}<1$ ).

\section{Discussion}

The data from the yes-no recognition test revealed a DF effect, indicating compliance with the $\mathrm{R}$ and $\mathrm{F}$ memory instructions. Given that this was the case, the question of main interest was whether these memory instructions would interact with the motoric form of IOR. Participants responded to a target by making a saccade to its location. We observed an F> $\mathrm{R}$ IOR pattern in the saccadic RTs, demonstrating an interaction of memory instruction with the motoric form of IOR. This is consistent with the results of Taylor and Fawcett (2011; see also Thompson et al., 2014), who concluded that a memory instruction interacts selectively with the motoric form of IOR.

To fully test Taylor and Fawcett's (2011) conclusions about the F> R IOR difference, it was necessary to explicitly test whether memory instruction would also interact with the visual form of IOR. This was done in Experiment 2.

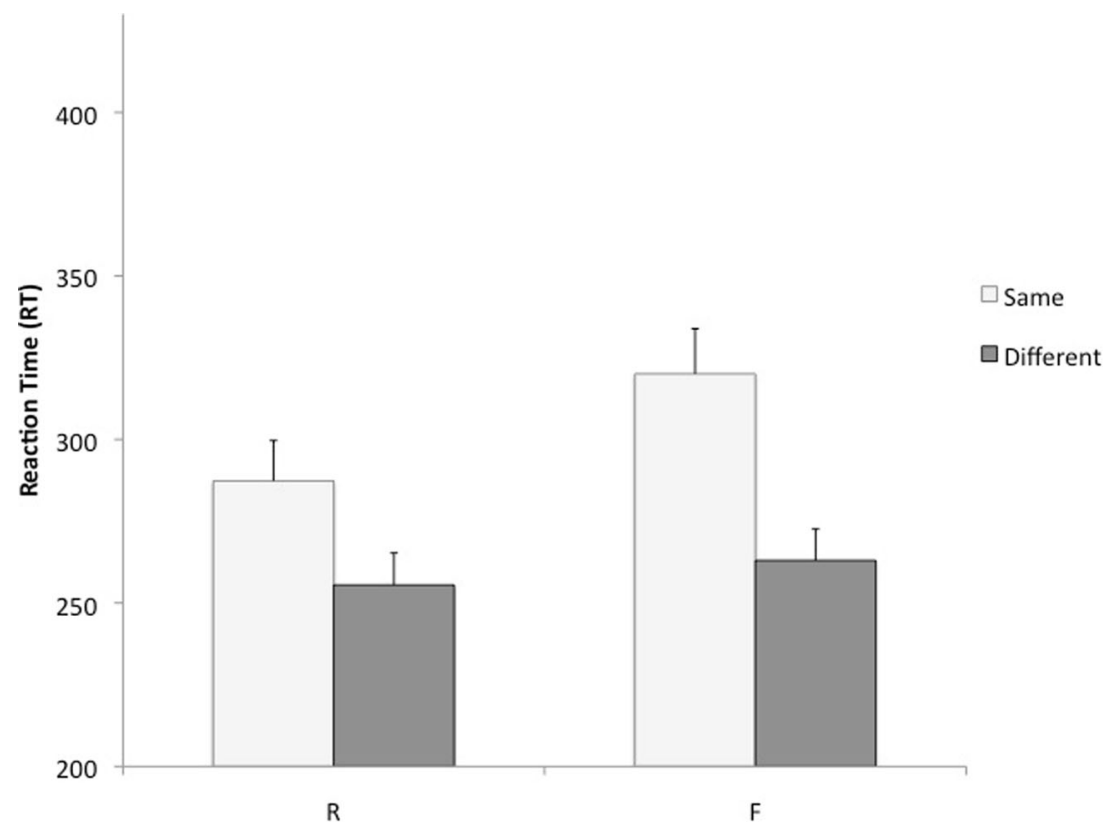

Fig. 2 Mean RTs, in milliseconds, after R and F memory instructions to targets appearing in the same location as the word versus the different location in Experiment 1. Error bars are standard errors 


\section{Experiment 2}

In Experiment 2, participants were presented with study words one at a time to the left or the right of central fixation, each followed by an auditory R or F memory instruction, and then by a target in the same location as the word or in the opposite one. Participants maintained fixation throughout the entire trial and localized the target with a manual button-press. Because the oculomotor system was suppressed, IOR in this condition should be visual in nature (Chica et al., 2010b; Hilchey et al., 2012; Hunt \& Kingstone, 2003; Taylor \& Klein, 2000). To reiterate our predictions: If memory instruction selectively interacts with motoric IOR, the F> R IOR pattern should not occur in this experiment; if memory instruction also interacts with visual IOR, the F $>$ R IOR pattern should occur here.

\section{Method}

Participants Twenty-seven ${ }^{2}$ participants were recruited from the undergraduate subject pool at Dalhousie University and received one credit point for participating. All of the participants reported normal or corrected-to-normal vision and a good understanding of the English language. The experiment was approved by the Human Research Ethics board at Dalhousie University, and thus meets the ethical standards set forth in the Tri-Council Policy Statement.

Materials The materials were the same as in Experiment 1, but included the use of a Universal Serial Bus keyboard to record manual responses.

Procedure The procedure was identical to that in Experiment 1, with the exception of the response required to the target. Instead of making a saccade to the target, participants were told to maintain fixation at screen center throughout the entire trial. When the target appeared on the left, they were to press the " $\mathrm{f}$ " key, and when it appeared on the right, they were to press the " $\mathrm{j}$ " key. Study trials were aborted if erroneous eye movements were made at any time during the trial.

\footnotetext{
${ }^{2}$ Note that for Experiment 2 we recruited two fewer participants than for Experiment 1. This was due to variations in participant volunteer rates between the experiments. To ensure that the larger sample size in Experiment $1(n=29)$ than in Experiment $2(n=27)$ did not affect our conclusions, we repeated the analysis of the Experiment 1 data after excluding the last two participants to join $(n=27)$; the pattern of results was unchanged.
}

\section{Results}

Proportion of retained study trials Study trials were retained for analysis only if participants refrained from making eye movements after the start of the trial. To determine whether study trials were retained differentially across conditions, a 2 (Word-Target Location: same, different) $\times 2$ (Memory Instruction: $\mathrm{R}, \mathrm{F}$ ) repeated measures ANOVA was conducted with the proportions of retained trials as the dependent measure. Descriptive statistics can be found in Table 2. There were no significant effects or interactions (all $F \mathbf{s}<1$ ). Thus, participants' ability to follow the fixation instructions was not influenced by the type of trial that was presented.

Recognition accuracy Although words from aborted study trials were tested during the recognition phase, they were excluded from the calculations of recognition accuracy. The data from the recognition test were analyzed using a one-way repeated measures ANOVA with word type (R, F, foil) as the independent variable and the proportion of "yes" responses as the dependent variable. We found a significant main effect of word type $[F(2,52)=79.467, M S E=.006, p<.001]$, such that $\mathrm{R}$ items $(M=.45)$ were recognized at a higher rate than $\mathrm{F}$ items $(M=.33)[t(26)=6.063, p<.001]$. This is the expected $\mathrm{DF}$ effect (better memory for $\mathrm{R}$ than for $\mathrm{F}$ items). Both $\mathrm{R}$ and $\mathrm{F}$ items were recognized at a higher rate than foil words $(M=$ .18) $[t(26)=10.499, p<.001$, and $t(26)=8.120, p<.001$, respectively]. These results confirmed that participants had used the memory instructions as intended at study.

Manual RTs Given that participants had used the memory instructions as intended, the key question was whether these instructions would interact with the IOR effect measured by manual RTs to the targets presented at study. See Fig. 3 for descriptive statistics. A 2 (Word-Target Location: same, different) $\times 2$ (Memory Instruction: R, F) repeated measures ANOVA was conducted on the manual RTs to the targets. A significant main effect of word-target location was apparent $[F(1,26)=19.537, M S E=655.255, p<.001]$, with slower RTs to targets in the same location as the previous word than to those in the other location (an IOR effect). The main effect of memory instruction was not significant $(F<1)$. Finally, a

Table 2 Descriptive statistics of the proportions of retained study trials per condition in Experiment 2. Means are reported, with standard deviations in parentheses

\begin{tabular}{lll}
\hline & \multicolumn{2}{l}{ Word-Target Location } \\
\cline { 2 - 3 } Memory Instruction & Same & Different \\
\hline Remember & $.71(.14)$ & $.69(.16)$ \\
Forget & $.69(.15)$ & $.70(.13)$ \\
\hline
\end{tabular}




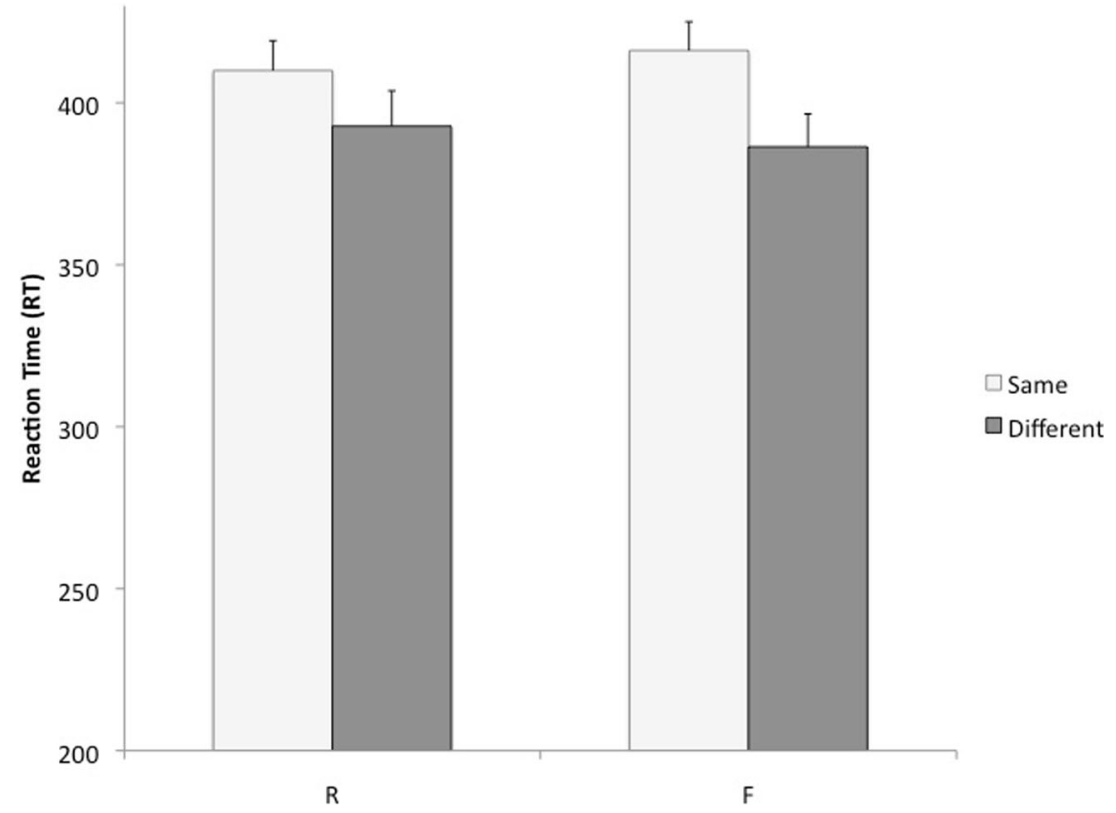

Fig. 3 Mean RTs, in milliseconds, after R and F memory instructions to targets appearing in the same location as the word versus the different location in Experiment 2. Error bars are standard errors

significant Word-Target Location $\times$ Memory Instruction interaction $[F(1,26)=4.744, M S E=263.426, p=.039]$ was due to the fact that the magnitude of IOR (same RT- different RT) was greater after $\mathrm{F}(M=28.578 \mathrm{~ms})$ than after $\mathrm{R}(M=$ $14.971 \mathrm{~ms})$ instructions.

Analogous analyses on the accuracy of target responses yielded no significant effects (all $F_{\mathrm{s}}<1$ ).

\section{Discussion}

The data from the yes-no recognition test revealed a DF effect, indicating compliance with the $\mathrm{R}$ and $\mathrm{F}$ memory instructions. Given that this was the case, the question of main interest was whether these memory instructions would interact with the visual form of IOR. We observed the F> R IOR pattern in the analysis of the manual RTs to the study trial targets, demonstrating an interaction of memory instruction with the visual form of IOR. This is inconsistent with the results of Taylor and Fawcett (2011; see also Thompson et al., 2014), who concluded that memory instructions interact selectively with the motoric form of IOR.

Although the results of the present experiment are inconsistent with those of Taylor and Fawcett (2011), this is perhaps not surprising, given the potential confound that we outlined above. Since participants were making saccades to each word in Taylor and Fawcett (2011), the visual form of IOR that might be expected to emerge in their perceptual discrimination task would have been masked (Chica et al., 2010b). However, we thought it prudent to replicate and extend the results of the present experiment by testing for an interaction of memory instruction and visual IOR using the same perceptual discrimination response used by Taylor and Fawcett (2011), while restricting eye movements as in the present experiment. This would give us more confidence in our conclusion that memory instructions interact with both forms of IOR.

\section{Experiment 3}

The results of Experiment 2 conflict with a previous conclusion from Taylor and Fawcett (2011). In one of their experiments, participants were presented with a word to the left or the right, followed by an auditory R or F instruction, and then by a triangle to the left or the right. Participants were required to indicate with a button-press whether the triangle was upright or inverted. Because Taylor and Fawcett (2011) found no significant interaction between memory instruction and IOR for this perceptual discrimination task, they concluded that memory instructions interact only with the motoric form of IOR, and not with the visual form. However, this conclusion was based on the results of experiments that neither restricted nor monitored eye movements. And, as we noted previously, visual IOR does not occur when the eyes are unrestrained (Chica et al., 2010b). This suggests that the lack of eye movement monitoring likely undermined Taylor and Fawcett's (2011) ability to find an interaction of memory instruction with the visual form of IOR. Perhaps an F instruction leads not only to a bias against responding to targets that arise at the location of the previous misinformation, but, in the 
absence of eye movements, also to a perceptual processing deficit for targets presented at that location. If the conclusions of Experiment 2 are correct, and memory instructions do interact with visual IOR, then prohibiting and monitoring eye movements in a replication of Taylor and Fawcett's (2011) perceptual discrimination task should produce the F> R IOR pattern that Taylor and Fawcett (2011) could not. To test this, in Experiment 3 we presented participants with a word to the left or the right, followed by an auditory R or F instruction, and then by a triangular target to the left or the right. Participants were required to discriminate between upright and inverted triangles with a manual button-press while maintaining fixation at screen center throughout the trial. Eye movement monitoring ensured that participants complied with the instruction to refrain from making movements.

\section{Method}

Participants Thirty-five $\mathrm{e}^{3}$ participants were recruited from the undergraduate subject pool at Dalhousie University and received one credit point for participating. All of the participants reported normal or corrected-to-normal vision and a good understanding of the English language. The experiment was approved by the Human Research Ethics board at Dalhousie University, and thus meets the ethical standards set forth in the Tri-Council Policy Statement.

Materials The materials used here were identical to those used in Experiments 1 and 2, with the exception of the targets. Yellow triangles (of the same size and eccentricity as the gray placeholders) now served as the targets.

Procedure The procedure used was identical to that of Experiment 2, with the following exceptions. A triangular target was presented on each trial, instead of a circular target. When the target appeared, participants were required to press the " $\mathrm{f}$ " key with the index finger of their left hand if the triangle was upright (i.e., pointing upward), or the "j" key with the index finger of their right hand if the triangle was inverted (i.e., pointing downward).

\footnotetext{
${ }^{3}$ Note that the sample size for Experiment 3 was greater than those of both Experiments 1 and 2. This was motivated by the fact that Experiment 3 was an attempt to replicate Taylor and Fawcett's (2011) discrimination experiment, which for them produced a null result. Given this, we wished to ensure that we had enough power to observe a potentially small effect.
}

\section{Results}

Proportion of retained study trials Study trials were retained for analysis only if participants refrained from making eye movements after the start of the trial. To determine whether study trials were retained differentially across conditions, a 2 (Word-Target Location: same, different) $\times 2$ (Memory Instruction: $\mathrm{R}, \mathrm{F}$ ) repeated measures ANOVA was conducted with the proportions of retained trials as the dependent measure. Descriptive statistics can be found in Table 3. There was a significant main effect of word-target location $[F(1,34)=$ $5.172, M S E=.003, p=.029]$, with a higher proportion of retained trials when the target appeared in the same location as the word than when it appeared in the different location. No other effects were significant (all $F_{\mathrm{s}}<1$ ).

Recognition accuracy Although words from aborted study trials were tested during the recognition phase, they were excluded from the calculations of recognition accuracy. The data from the recognition test were analyzed using a one-way repeated measures ANOVA with word type (R, F, foil) as the independent variable and the proportion of "yes" responses as the dependent variable. We found a significant main effect of word type $[F(2,68)=91.668, M S E=.010, p<.001]$, such that $\mathrm{R}$ items $(M=.46)$ were recognized at a higher rate than $\mathrm{F}$ items $(M=.33)[t(34)=5.547, p<.001]$. This is the expected $\mathrm{DF}$ effect (better memory for $\mathrm{R}$ than for $\mathrm{F}$ items). Both $\mathrm{R}$ and $\mathrm{F}$ items were recognized at a higher rate than foil words $(M=$ .13) $[t(34)=11.583, p<.001$, and $t(34)=9.492, p<.001$, respectively]. These results confirmed that participants had used the memory instructions as intended at study.

Discrimination RTs Given that participants had used the memory instructions as intended, the key question was whether these instructions would interact with the IOR effect measured by RTs to discriminate the target triangle on study trials. See Fig. 4 for descriptive statistics. A 2 (Word-Target Location: same, different) $\times 2$ (Memory Instruction: R, F) repeated measures ANOVA was conducted on the discrimination RTs. A significant main effect of word-target location emerged $[F(1,34)=4.921, M S E=1224.310, p<.033]$, with slower RTs to targets in the same location as the previous word

Table 3 Descriptive statistics of the proportions of retained study trials per condition in Experiment 3. Means are reported, with standard deviations in parentheses

\begin{tabular}{lll}
\hline & \multicolumn{2}{l}{ Word-Target Location } \\
\cline { 2 - 3 } Memory Instruction & Same & Different \\
\hline Remember & $.79(.14)$ & $.77(.12)$ \\
Forget & $.80(.10)$ & $.78(.13)$ \\
\hline
\end{tabular}




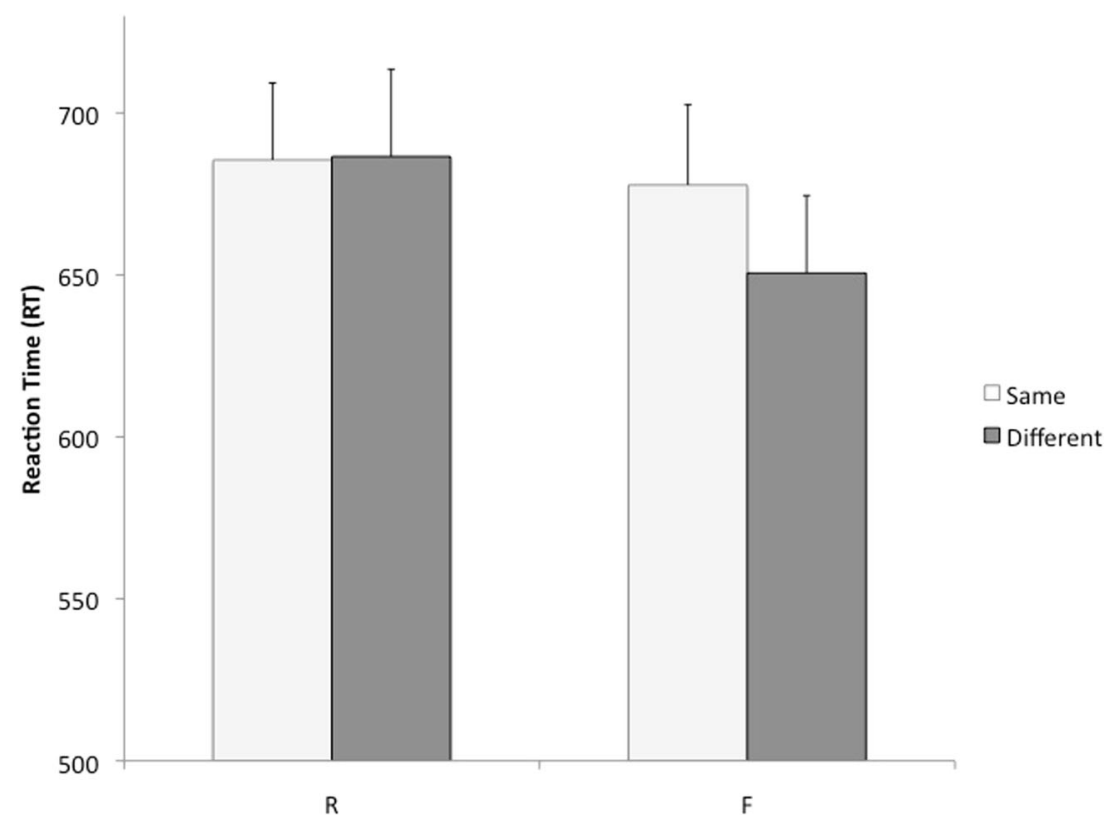

Fig. 4 Mean RTs, in milliseconds, after R and F memory instructions to targets appearing in the same location as the word versus the different location in Experiment 3. Error bars are standard errors

than to those in the other location (an IOR effect). We also found a significant main effect of memory instruction $[F(1$, $34)=11.157, M S E=1505.005, p=.002]$, with slower RTs after $\mathrm{R}$ than after $\mathrm{F}$ instructions. Finally, a significant WordTarget Location $\times$ Memory Instruction interaction $[F(1,34)=$ 6.792, $M S E=1032.661, p=.013]$ was due to the fact that the magnitude of IOR (same RT - different RT) was greater after F $(M=27.277 \mathrm{~ms})$ than after $\mathrm{R}(M=-1.035 \mathrm{~ms})$ instructions.

Analogous analyses on the accuracy of target responses yielded no significant effects (all $F_{\mathrm{S}}<1$ ).

\section{Discussion}

The results from the yes-no recognition test confirmed a DF effect, suggesting that participants complied with the $\mathrm{R}$ and $\mathrm{F}$ instructions. Given that this was the case, the critical question was whether instituting eye movement monitoring in a replication of Taylor and Fawcett's (2011) perceptual discrimination task would reveal the interaction of memory instruction and visual IOR that Taylor and Fawcett had discounted. Indeed, it did; Experiment 3 revealed an F > R IOR pattern for the same perceptual discrimination task employed by Taylor and Fawcett (2011). In so doing, the results of Experiment 3 bolstered the conclusion drawn from the results of Experiments 1 and 2: Memory instructions interact not only with the motoric form of IOR, but also with the visual form.

Before proceeding to the General Discussion, it is worth noting that in Experiment 3, the larger IOR effect for $\mathrm{F}$ than for $\mathrm{R}$ trials reflected a significant IOR effect for the former and not for the latter condition. This is consistent with previous investigations of IOR and directed forgetting, in which the trend was that in some cases the IOR effect would not be significant after $\mathrm{R}$ instructions, but even in the cases in which significant IOR was observed after $\mathrm{R}$ instructions, the magnitude of IOR would be greater after $\mathrm{F}$ than after $\mathrm{R}$ instructions. That is, the increased magnitude of IOR after F instructions is always observed, and this is sometimes accompanied by a nonsignificant IOR effect after R items (Taylor, 2005; Taylor \& Fawcett, 2011; Thompson et al., 2014). This relative increase in magnitude of IOR after $\mathrm{F}$ instructions and decrease after $\mathrm{R}$ instructions has been made in comparison to a nomemory control condition (Taylor, 2005; Taylor \& Fawcett, 2011). The decreased magnitude of IOR after R instructions may be due to individual differences in study strategies (perhaps explaining its inconsistent appearance), and likely indicates attentional dwelling on $\mathrm{R}$ items to aid in elaborative encoding; this would result in a delay in the appearance of IOR, which would be caused by the onset of the word, but masked by facilitatory effects until attention was withdrawn (Danziger \& Kingstone, 1999).

\section{General discussion}

IOR can be conceived of as a mechanism that facilitates visual search for novelty (Klein, 2000; Klein \& MacInnes, 1999; MacInnes \& Klein, 2003). After attention is captured by a particular stimulus, reinspection of that location is prevented in one of two ways, depending on the state of the oculomotor system (Chica et al., 2010b; Hilchey et al., 2012; Hunt \& 
Kingstone, 2003; Taylor \& Klein, 2000). When the oculomotor system is active, IOR reflects a motoric bias against making responses toward the cued location. When the oculomotor system is inactive or suppressed, IOR reflects a perceptual processing deficit at the stimulus location.

We observed effects of memory instruction on both of these forms of IOR. It seems unlikely that memory instruction has two entirely independent effects, one that interacts only with a motor response bias and one that interacts only with perceptual processing. Instead, it is more parsimonious to assume that, despite the fact that motoric and visual IOR represent different behavioral manifestations of the aftereffects of peripheral visual stimulation (Chica et al., 2010b; Hilchey et al., 2012; Hunt \& Kingstone, 2003; Taylor \& Klein, 2000), they must have upstream processing in common, and it is this common upstream processing that interacts with the memory instruction.

Most research on the two forms of IOR has focused on the mechanisms that differentiate them. However, they may share some commonalities. For example, in an rTMS study, Bourgeois et al. (2013) found that disruption of the intraparietal sulcus (IPS) disrupted both motoric and visual IOR for left-side targets. Thus, the IPS may represent a neural correlate of both motoric and visual IOR. In attention research, the IPS is known as the seat of a spatial salience map in which the salience of environmental stimuli is represented and can be modified on the basis of experience (Silver \& Kastner, 2009; van Koningsbruggen, Gabay, Sapir, Henik, \& Rafal, 2010). In the IOR framework, it is thought that the salience of a cued location in the IPS is diminished/inhibited in order to allow orienting to new spatial locations, causing increased RTs to targets presented in the cued location (Sapir, Hayes, Henik, Danziger, \& Rafal, 2004; Vivas, Humphreys, \& Fuentes, 2006). In accordance with the idea that this area could be upstream of both the motoric and visual forms of IOR, the IPS has connections to the superior colliculus (SC, known to be involved in motoric IOR: Anderson \& Rees, 2011; Bourgeois et al., 2012; Bourgeois et al., 2013; Dorris, Klein, Everling, \& Munoz, 2002; Robinson, Bowman, \& Kertzman, 1995). Not only this, but the dorsal parieto-frontal network that encompasses the IPS is also tightly linked with the ventral parietofrontal network that encompasses the temporo-parietal junction (TPJ, known to be involved in visual IOR: Asplund, Todd, Snyder, \& Marois, 2010; Bourgeois et al., 2012; Bourgeois et al., 2013). Interestingly, research has suggested that the salience map in the IPS has much broader applications than mapping the salience of environmental spatial locations. Rather, the salience map is thought to be involved with guiding top-down attention not only spatially, but also with respect to particular target features or semantic associations, and even with guiding the retrieval of target memories (Cabeza, 2008; Ciaramelli et al., 2008; Silver \& Kastner, 2009). Although this account is highly speculative, it introduces the intriguing possibility that memory instructions interact with motoric and visual IOR effects by altering representations within the IPS saliency map. Essentially, the locations of to-be-forgotten items become relatively less salient than those that contained to-be-remembered items.

Although fMRI research on directed forgetting has focused on frontal and medial temporal lobe activation, parietal activation is often associated with instantiating an instruction to forget at study (e.g., Bastin et al. 2012; Nowicka et al., 2011; Saletin et al., 2011; Wylie et al., 2008). In addition, electrophysiological investigations of directed forgetting have consistently shown that $\mathrm{R}$ instructions are associated with a parietally distributed positivity that is absent after $\mathrm{F}$ instructions (Hauswald, Schulz, Iordanov, \& Kissler, 2011; Hsieh, Hung, Tzeng, Lee, \& Cheng, 2009; Lin, Kuo, Liu, Han, \& Cheng, 2013; Paz-Caballero, Menor, \& Jiménez, 2004; Ullsperger, Mecklinger, \& Muller, 2000; van Hooff \& Ford, 2011). This could represent parietal inhibition after $F$, but not $\mathrm{R}$, instructions. When combined with an IOR cueing paradigm, this inhibition after $\mathrm{F}$ items might be additive with that observed due to IOR. Finally, parietal areas, including the IPS, have been found to be associated with the suppression of unwanted memories in other experimental paradigms, such as think/no-think (Anderson et al., 2004). So, although the present results certainly are not able to directly support the hypothesis that activation in the IPS in particular may be influenced by memory instructions, this is one possibility that is consistent with the existing IOR and directed forgetting literatures.

Collectively, the research on IOR in directed forgetting has taught us much about the cognitive consequences of instantiating an intention to forget. We now have a substantial amount of evidence that attention is more readily withdrawn after $\mathrm{F}$ than after R instructions (Fawcett \& Taylor, 2010; Taylor, 2005; Taylor \& Fawcett, 2011; Thompson et al., 2014; the present experiments). This differential withdrawal of attention helps direct cognitive resources away from unwanted information and toward relevant information. Withdrawing attention is a cognitively demanding process, which results in the reduced availability of cognitive resources following $\mathrm{F}$ as compared to $\mathrm{R}$ instructions (Cheng, Liu, Lee, Hung, \& Tzeng, 2012; Fawcett \& Taylor, 2008). We know that, although there is conflicting evidence regarding whether the $\mathrm{F}>$ $\mathrm{R}$ IOR difference is related to the magnitude of the DF effect (Fawcett \& Taylor, 2010; Taylor \& Fawcett, 2011; Thompson et al., 2014), successful instantiation of an $\mathrm{F}$ instruction is related to the availability of cognitive resources: Forgetting is more successful under highly demanding task conditions (Lee, 2012; Lee \& Lee, 2011). Thus, the differential withdrawal of attention may be directly beneficial for successful intentional forgetting, by redirecting attention away from unwanted information. It may also indirectly benefit successful intentional forgetting by occupying cognitive resources. 
Not only does an F instruction cause attention to be differentially withdrawn, it also has lasting and wide-ranging consequences on subsequent information processing. The present experiment has shown that memory instructions interact with IOR in a way that enhances both a bias against responding to the source of the $\mathrm{F}$ item and perceptual impairments at that source. IOR has been conceptualized as a mechanism that facilitates visual search by encouraging the inspection of novel, uninspected locations (Klein, 2000; Klein \& MacInnes, 1999; MacInnes \& Klein, 2003). In this case, a location is inspected and found to be irrelevant, so processing and responses are directed away from that location in order to avoid the constant reinspection of a known irrelevant source. The magnification of this difference by an $\mathrm{F}$ instruction is a logical extension. Previous research has suggested that instantiating an $\mathrm{F}$ instruction results not only in decreased memory for $\mathrm{F}$ items, but also in impoverished encoding of contextual/ episodic information presented in close temporal proximity to the F item (Fawcett \& Taylor, 2012; Hourihan, Goldberg, \& Taylor, 2007). Fawcett and Taylor (2012) showed decreased memory for probe words presented after $\mathrm{F}$ as compared to after $\mathrm{R}$ instructions, suggesting that incidental memory for information presented soon after an $\mathrm{F}$ instruction is decreased. Hourihan et al., (2007) showed that presenting items in the same spatial location at study and test benefited memory performance only for $\mathrm{F}$ items, but not for $\mathrm{R}$ items. This suggests that the encoding of contextual characteristics (such as spatial location) was already strong for $\mathrm{R}$ items, but the weak encoding of such details for $\mathrm{F}$ items leads to a significant improvement in memory performance with the addition of such contextual cues at test. The notion that an F instruction disrupts the episodic encoding of the event is also supported by fMRI studies of item-method directed forgetting, in which instantiating an $\mathrm{F}$ instruction has been associated with frontal and medial temporal activation (Bastin et al., 2012; Nowicka et al., 2011; Saletin et al., 2011; Wylie et al., 2008) The F $>$ R IOR difference represents a mechanism by which this disruption of episodic encoding occurs. We have shown that an $\mathrm{F}$ instructions limits the degree to which contextual elements are able to capture attention, by modulating the perceptual processing of the event and/or the motor output (visual and motoric IOR). This would result in impoverished encoding of the event, and therefore reduced memory strength for $\mathrm{F}$ as compared to R items - the DF effect.

To conclude, we have shown that instantiating an instruction to forget increases the magnitude of IOR, leading to both a bias against responding to the $\mathrm{F}$ item source and perceptual decrements at the $\mathrm{F}$ item location, depending on the state of the oculomotor system. These consequences of instantiating an instruction to forget are adaptive, insofar as they promote the encoding of valid and relevant observations about the world and prevent the encoding of invalid, irrelevant observations.

\section{References}

Anderson, M. C., Bjork, R. A., \& Bjork, E. L. (1994). Remembering can cause forgetting: Retrieval dynamics in long-term memory. Journal of Experimental Psychology: Learning, Memory, and Cognition, 20, 1063-1087. doi:10.1037/0278-7393.20.5.1063

Anderson, M. C., \& Neely, J. H. (1996). Interference and inhibition in memory retrieval. In E. L. Bjork \& R. A. Bjork (Eds.), Memory (Handbook of Perception and Cognition (2nd ed., pp. 237-313). San Diego, CA: Academic Press.

Anderson, M. C., Ochsner, K. N., Kuhl, B., Cooper, J., Robertson, E., Gabrieli, S. W., \& Gabrieli, J. D. E. (2004). Neural systems underlying the suppression of unwanted memories. Science, 303, 232235. doi:10.1126/science.1089504

Anderson, E. J., \& Rees, G. (2011). Neural correlates of spatial orienting in the human superior colliculus. Journal of Neurophysiology, 106, 2273-2284.

Asplund, C. L., Todd, J. J., Snyder, A. P., \& Marois, R. (2010). A central role for the lateral prefrontal cortex in goal-directed and stimulus driven attention. Nature Neuroscience, 13, 507-512.

Basden, B. H., \& Basden, D. R. (1998). Directed forgetting: A contrast of methods and interpretations. In J. M. Golding \& C. M. MacLeod (Eds.), Intentional forgetting: Interdisciplinary approaches (pp. 139-172). Mahwah, NJ: Erlbaum.

Bastin, C., Feyers, D., Majerus, S., Balteau, E., Degueldre, C., Luxen, A., \& Collette, F. (2012). The neural substrates of memory suppression: A fMRI exploration of directed forgetting. PLoS ONE, 7, e29905. doi:10.1371/journal.pone.0029905

Bjork, R. A., \& Woodward, A. E. (1973). Directed forgetting of individual words in free recall. Journal of Experimental Psychology, 99, $22-27$.

Bourgeois, A., Chica, A. B., Migliaccio, R., Thiebault de Schotten, M., \& Bartolomeo, P. (2012). Cortical control of inhibition of return: Evidence from patients with inferior parietal damage and visual neglect. Neuropsychologia, 50, 800-809. doi:10.1016/j. neuropsychologia.2012.01.014

Bourgeois, A., Chica, A. B., Valero-Cabré, A., \& Bartolomeo, P. (2013). Cortical control of inhibition of return: Causal evidence for taskdependent modulations by dorsal and ventral parietal regions. Cortex, 49, 2229-2238.

Cabeza, R. (2008). Role of parietal regions in episodic memory retrieval: The dual attentional processes hypothesis. Neuropsychologia, 46, 1813-1827.

Cheng, S. K., Liu, I., Lee, J. R., Hung, D. L., \& Tzeng, O. J.-L. (2012). Intentional forgetting might be more effortful than remembering: An ERP study of item-method directed forgetting. Biological Psychology, 89, 283-292.

Chica, A. B., Klein, R. M., Rafal, R. D., \& Hopfinger, J. B. (2010a). Endogenous saccade preparation does not produce inhibition of return: Failure to replicate Rafal, Calabresi, Brennan, \& Sciolto (1989). Journal of Experimental Psychology: Human Perception and Performance, 36, 1193-1206. doi:10.1037/a0019951

Chica, A. B., Taylor, T. L., Lupiáñez, J., \& Klein, R. M. (2010b). Two mechanisms underlying inhibition of return. Experimental Brain Research, 201, 25-35. doi:10.1007/s00221-009-2004-1

Ciaramelli, E., Grady, C. L., \& Moscovitch, M. (2008). Top-down and bottom-up attention to memory: A hypothesis (AtoM) on the role of the posterior parietal cortex in memory retrieval. Neuropsychologia, 46, 1828-1851.

Conway, M. A. (2009). Episodic memories. Neuropsychologia, 47, 2305-2313. doi:10.1016/j.neuropsychologia.2009.02.003

Danziger, S., \& Kingstone, A. (1999). Unmasking the inhibition of return phenomenon. Perception \& Psychophysics, 61, 1024-1037. doi:10. 3758/BF03207610 
Dorris, M. C., Klein, R. M., Everling, S., \& Munoz, D. P. (2002). Contribution of the primate superior colliculus to inhibition of return. Journal of Cognitive Neuroscience, 14, 1256-1263. doi:10. $1162 / 089892902760807249$

Duncan, J., \& Humphreys, G. W. (1989). Visual search and stimulus similarity. Psychological Review, 96, 433-458. doi:10.1037/0033295X.96.3.433

Fawcett, J. M., \& Taylor, T. L. (2008). Forgetting is effortful: Evidence from reaction time probes in an item-method directed forgetting task. Memory \& Cognition, 36, 1168-1181. doi:10.3758/MC.36.6. 1168

Fawcett, J. M., \& Taylor, T. L. (2010). Directed forgetting shares mechanisms with attentional withdrawal but not with stop-signal inhibition. Memory \& Cognition, 38, 797-808. doi:10.3758/MC.38.6.797

Fawcett, J. M., \& Taylor, T. L. (2012). The control of working memory resources in intentional forgetting: Evidence from incidental probe word recognition. Acta Psychologica, 139, 84-90.

Gallagher, S. (2000). Philosophical conceptions of the self: Implications for cognitive science. Trends in Cognitive Sciences, 4, 14-21. doi: 10.1016/S1364-6613(99)01417-5

Hauswald, A., Schulz, H., Iordanov, T., \& Kissler, J. (2011). ERP dynamics underlying successful directed forgetting of neutral but not negative pictures. Social Cognitive and Affective Neuroscience, 6 , 450-459.

Henderickx, D., Maetens, K., \& Soetens, E. (2012). The involvement of bottom-up saliency processing in endogenous inhibition of return. Attention, Perception, \& Psychophysics, 74, 285-299.

Hilchey, M. D., Klein, R. M., \& Ivanoff, J. (2012). Perceptual and motor inhibition of return: Components or flavors? Attention, Perception, \& Psychophysics, 74, 1416-1429. doi:10.3758/s13414-012-0332-x

Hourihan, K. L., Goldberg, S., \& Taylor, T. L. (2007). The role of spatial location in remembering and forgetting peripheral words. Canadian Journal of Experimental Psychology, 61, 91-101.

Hourihan, K. L., Ozubko, J. D., \& MacLeod, C. M. (2009). Directed forgetting of visual symbols: Evidence for nonverbal selective rehearsal. Memory \& Cognition, 37, 1059-1068. doi:10.3758/MC.37. 8.1059

Hsieh, L.-T., Hung, D. L., Tzeng, O. J.-L., Lee, J. R., \& Cheng, S.-K. (2009). An event-related potential investigation of the processing of Remember/Forget cues and item encoding in item-method directed forgetting. Brain Research, 1250, 190-201. doi:10.1016/j.brainres. 2008.11.016

Hunt, A. R., \& Kingstone, A. (2003). Inhibition of return: Dissociating attentional and occulomotor components. Journal of Experimental Psychology: Human Perception and Performance, 29, 1068-1074. doi:10.1037/0096-1523.29.5.1068

Klein, R. M. (2000). Inhibition of return. Trends in Cognitive Sciences, 4, 138-147. doi:10.1016/S1364-6613(00)01452-2

Klein, R. M., \& MacInnes, W. J. (1999). Inhibition of return is a foraging facilitator in visual search. Psychological Science, 10, 346-352. doi: 10.1111/1467-9280.00166

Lee, Y.-S. (2012). Cognitive load hypothesis of item-method directed forgetting. Quarterly Journal of Experimental Psychology, 65, $1110-1122$

Lee, Y.-S., \& Lee, H.-M. (2011). Divided attention facilitated intentional forgetting: Evidence from item-method directed forgetting. Consciousness and Cognition, 20, 618-626.

Lin, W.-J., Kuo, Y.-C., Liu, T.-L., Han, Y.-J., \& Cheng, S.-K. (2013). Intentional forgetting reduces the semantic processing of to-beforgotten items: An ERP study of item-method directed forgetting. Psychophysiology, 50, 1120-1132.

MacInnes, W. J., \& Klein, R. M. (2003). Inhibition of return biases orienting during the search of complex scenes. Scientific World Journal, 3, 75-86.

MacLeod, C. M. (1975). Long-term recognition and recall following directed forgetting. Journal of Experimental Psychology:
Human Learning and Memory, 1, 271-279. doi:10.1037/ 0278-7393.1.3.271

MacLeod, C. M. (1998). Directed forgetting. In J. M. Golding \& C. M. MacLeod (Eds.), Intentional forgetting: Interdisciplinary approaches (pp. 1-57). Mahwah, NJ: Erlbaum.

MacLeod, C. M. (1999). The item and list methods of directed forgetting: Test differences and the role of demand characteristics. Psychonomic Bulletin \& Review, 6, 123-129. doi:10.3758/ BF03210819

Nowicka, A., Marchewka, A., Jednorog, K., Tacikowski, P., \& Brechmann, A. (2011). Forgetting of emotional information is hard: An fMRI study of directed forgetting. Cerebral Cortex, 21, 539549.

Paz-Caballero, M. D., Menor, J., \& Jiménez, J. M. (2004). Predictive validity of event-related potentials (ERPs) in relation to the directed forgetting effects. Clinical Neurophysiology, 115, 369-377.

Posner, M. I. (1980). Orienting of attention. Quarterly Journal of Experimental Psychology, 32, 3-25. doi:10.1080/ 00335558008248231

Posner, M. I., \& Cohen, Y. (1984). Components of visual orienting. In H. Bouma \& D. G. Bouwhuis (Eds.), Attention and performance X: Control of language processes (pp. 531-556). Hillsdale, NJ: Erlbaum.

Posner, M. I., \& Petersen, S. E. (1990). The attention system of the human brain. Annual Reviews of Neuroscience, 13, 25-42. doi:10.1146/ annurev.ne.13.030190.000325

Postman, L., \& Underwood, B. J. (1973). Critical issues in interference theory. Memory \& Cognition, 1, 19-40.

Quinlan, C. K., Taylor, T. L., \& Fawcett, J. M. (2010). Directed forgetting: Comparing pictures and words. Canadian Journal of Experimental Psychology, 64, 41-46.

Rafal, R. D., Calabresi, P. A., Brennan, C. W., \& Sciolto, T. K. (1989). Saccade preparation inhibits reorienting to recently attended locations. Journal of Experimental Psychology: Human Perception and Performance, 15, 673-685. doi:10. 1037/0096-1523.15.4.673

Rayner, K. (1998). Eye movements in reading and information processing: 20 years of research. Psychological Bulletin, 124, 372-422. doi: 10.1037/0033-2909.124.3.372

Robinson, D. L., Bowman, E. M., \& Kertzman, C. (1995). Covert orienting of attention in macaques: II Contributions of parietal cortex. Journal of Neurophysiology, 74, 698-712.

Saletin, J. M., Goldstein, A. N., \& Walker, M. P. (2011). The role of sleep in directed forgetting and remembering of human memories. Cerebral Cortex, 21, 2534-2541.

Sapir, A., Hayes, A., Henik, A., Danziger, S., \& Rafal, R. (2004). Parietal lobe lesions disrupt saccadic remapping of inhibitory location tagging. Journal of Cognitive Neuroscience, 16, 503-509.

Satel, J., Hilchey, M. D., Wang, Z., Story, R., \& Klein, R. M. (2013). The effects of ignored versus foveated cues upon inhibition of return: An event-related potential study. Attention, Perception, \& Psychophysics, 75, 29-40. doi:10.3758/s13414-012-0381-1

Silver, M. A., \& Kastner, S. (2009). Topographic maps in human frontal and parietal cortex. Trends in Cognitive Sciences, 13, 488-495.

Taylor, T. L. (2005). Inhibition of return following instructions to remember and forget. Quarterly Journal of Experimental Psychology, 58, 613-629.

Taylor, T. L., \& Fawcett, J. M. (2011). Larger IOR effects following forget than following remember instructions depend on endogenous attentional withdrawal and target localization. Attention, Perception, \& Psychophysics, 73, 1790-1814. doi: 10.3758/s13414-011-0146-2

Taylor, T. L., \& Fawcett, J. M. (2012). Does an instruction to forget enhance memory for other presented items? Consciousness and Cognition, 21, 1186-1197. 
Taylor, T. L., \& Klein, R. M. (1998). On the causes and effects of inhibition of return. Psychonomic Bulletin \& Review, 5, 625-643. doi:10.3758/BF03208839

Taylor, T. L., \& Klein, R. M. (2000). Visual and motor effects in inhibition of return. Journal of Experimental Psychology: Human Perception and Performance, 26, 1639-1656. doi:10.1037/00961523.26.5.1639

Thompson, K. M., Fawcett, J. M., \& Taylor, T. L. (2011). Tag, you're it: Tagging as an alternative to yes/no recognition in item method directed forgetting. Acta Psychologica, 138, 171-175.

Thompson, K. M., Hamm, J. P., \& Taylor, T. L. (2014). Effects of memory instruction on attention and information processing: Further investigation of inhibition of return in item-method directed forgetting. Attention, Perception, \& Psychophysics, 76, 322-334. doi:10.3758/s13414-013-0584-0

Ullsperger, M., Mecklinger, A., \& Müller, U. (2000). An electrophysiological test of directed forgetting: The role of retrieval inhibition. Journal of Cognitive Neuroscience, 12, 924-940. van Hooff, J. C., \& Ford, R. M. (2011). Remember to forget: ERP evidence for inhibition in an item-method directed forgetting paradigm. Brain Research, 1392, 80-92. doi:10.1016/j.brainres.2011. 04.004

van Koningsbruggen, M. G., Gabay, S., Sapir, A., Henik, A., \& Rafal, R. D. (2010). Hemispheric asymmetry in the remapping and maintenance of visual saliency maps: A TMS study. Journal of Cognitive Neuroscience, 22, 1730-1738.

Vivas, A. B., Humphreys, G. W., \& Fuentes, L. J. (2006). Abnormal inhibition of return: A review and new data on patients with parietal lobe damage. Cognitive Neuropsychology, 23, 1049-1064. doi:10. 1080/02643290600588400

Woodward, A. E., Bjork, R. A., \& Jongeward, R. H. (1973). Recall and recognition as a function of primary rehearsal. Journal of Verbal Learning and Verbal Behavior, 12, 608-617.

Wylie, G. R., Foxe, J. J., \& Taylor, T. L. (2008). Forgetting as an active process: An FMRI investigation of item-method-directed forgetting. Cerebral Cortex, 18, 670-682. 